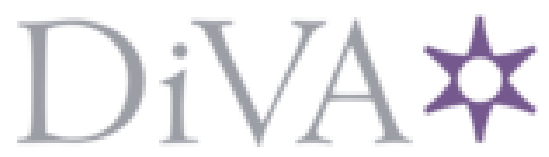

http://www.diva-portal.org

\title{
Postprint
}

This is the accepted version of a paper published in TIPS - Trends in Pharmacological Sciences. This paper has been peer-reviewed but does not include the final publisher proof-corrections or journal pagination.

Citation for the original published paper (version of record):

Matsson, P., Fenu, L., Lundquist, P., Wiśniewski, J., Kansy, M. et al. (2015)

Quantifying the impact of transporters on cellular drug permeability..

TIPS - Trends in Pharmacological Sciences, 35(5): 255-262

http://dx.doi.org/10.1016/j.tips.2015.02.009

Access to the published version may require subscription.

N.B. When citing this work, cite the original published paper.

Permanent link to this version:

http://urn.kb.se/resolve?urn=urn:nbn:se:uu:diva-248117 


\section{Quantifying the impact of transporters on cellular drug permeability}

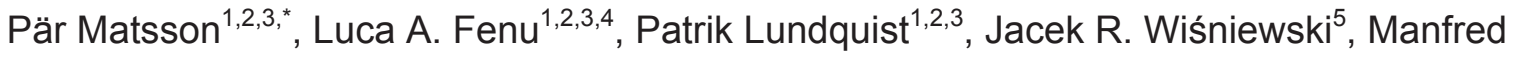
Kansy ${ }^{6}$ and Per Artursson ${ }^{1,2,3, *}$

${ }^{1}$ Department of Pharmacy, Uppsala University, Box 580, SE-751 23 Uppsala, Sweden

${ }^{2}$ Uppsala University Drug Optimization and Pharmaceutical Profiling Platform (UDOPP) - a node of the Chemical Biology Consortium Sweden

${ }^{3}$ Science for Life Laboratory Drug Discovery and Development Platform, Uppsala University, Sweden

${ }^{4}$ Present address: CADD group, Eli Lilly UK, Erl Wood Manor, Windlesham, GU20 6PH, UK

${ }^{5}$ Biochemical Proteomics Group, Department of Proteomics and Signal Transduction, MaxPlanck-Institute of Biochemistry, Am Klopferspitz 18, D-82152 Martinsried, Germany ${ }^{6}$ Roche Pharmaceutical Research and Early Development, Pharmaceutical Sciences, Roche Innovation Center Basel,

F. Hoffmann-La Roche Ltd, Grenzacherstrasse 124, 4070 Basel, Switzerland

*Address correspondence to:

Pär Matsson, PhD

Assistant Professor

Department of Pharmacy

Uppsala University

Box 580

SE-751 23 Uppsala, Sweden

Email: par.matsson@farmaci.uu.se

Phone: +46 - 184714630

Fax: $+46-184714223$
Per Artursson, PhD

Professor in Dosage Form Design

Department of Pharmacy

Uppsala University

Box 580

SE-751 23 Uppsala, Sweden

Email: per.artursson@farmaci.uu.se

Phone: +46 - 184714471

Fax: $+46-184714223$

\section{Keywords}

Drug transport; permeability; transporter; carrier-mediated transport; passive diffusion; systems biology 


\section{Abstract}

The conventional model of drug permeability has recently been challenged. An alternative model proposes that transporter-mediated flux is the sole mechanism of cellular drug permeation, instead of existing in parallel with passive transmembrane diffusion. We examined a central assumption of this alternative hypothesis; namely, that transporters can give rise to experimental observations that would typically be explained with passive transmembrane diffusion. Using systems-biology simulations based on available transporter kinetics and proteomic expression data, we found that such observations are possible in the absence of transmembrane diffusion, but only under very specific conditions that rarely or never occur for known human drug transporters. 


\section{Alternative models explaining cellular drug permeability}

The importance of transport proteins for the translocation of drugs across cell membranes has become increasingly appreciated over the past decades $[1,2]$. The predominant view of cellular drug transport is that such carrier-mediated transport coexists with diffusive transport across the lipid bilayers of cell membranes (Figure 1A) [3, 4]. The relative importance of carrier-mediated transport and transmembrane diffusion will then differ depending on: (i) the chemical structure of the transported molecule, which determines the molecule's interactions with transporters and membrane lipids; (ii) the local concentration of the transported drug, which determines the driving force for diffusion and the degree of saturation of carriermediated transport; and (iii) the panel of transporters available in the studied cell type (Box $1)$.

Recently, this predominant view of cellular drug transport has been challenged. An alternative model proposes that diffusion of drugs across lipid membranes is negligible in living cells and that transmembrane drug transport is more or less exclusively mediated by carrier proteins (transporters) (Figure 1A) [5]. The validity of the respective models ('coexistence' versus 'transporters-only') has been extensively debated [3-8]. The major tenet of the transporters-only model is that all observations of (putative) passive transmembrane diffusion are, instead, caused by one or more transporters. At first glance, the transportersonly model may appear impossible (or at least extremely daunting) to test: to exhaustively confirm the hypothesis, one would need to identify the missing carriers for all transported drug molecules. Here we present a systems-biology approach to probe some of the assumptions in the transporters-only model. Using simulations based on literature data on drug-transport kinetics and the expression levels of the transporter proteome, we explored the limits of carrier-mediated drug transport and to what extent putative transporters could explain observations of assumed passive transmembrane diffusion. 


\section{How efficient does a transporter need to be to account for observations of high drug permeabilities?}

Many drugs have high transcellular permeability (Box 2), either by design (e.g., to increase oral drug absorption and tissue distribution) or as a byproduct of the optimization of target affinity [3, 9-11]. Classical examples include propranolol and verapamil. These have permeability coefficients across Caco-2 intestinal epithelial cell monolayers (the most commonly used cellular barrier for permeability studies) in the range $200-1000 \times 10^{-6} \mathrm{~cm} / \mathrm{s}$ $[12,13]$. Notably, the transcellular permeabilities of these compounds are, to varying extents, limited by apical efflux transporters (Box 1), and their intrinsic membrane permeability (in the absence of efflux) can thus be even higher.

In comparison, how fast is carrier-mediated transport and what is the likelihood of observing rates on par with those observed for high-permeability drugs if the transport is carrier mediated only? To answer these questions, we combined transporter turnover numbers (kcat; Box 2) from the literature with recent data on the global proteomic expression of transporters in the Caco-2 intestinal epithelial cell line [14] (Figure 2). We thereby obtained realistic estimates of maximal carrier-mediated transport rates $\left(V_{\max }\right)$ for this extensively used cell permeability model $[11,15,16]$. We then compared the carrier-mediated rates that would be expected at a certain substrate concentration with rates calculated from experimentally determined permeability coefficients.

We used the facilitative glucose transporter 1 (GLUT1/SLC2A1) as an example of a high-capacity transporter. Intestinal glucose absorption and cellular glucose uptake are highly efficient processes $[17,18]$. This was apparent also from the collected parameters for GLUT1, which had the highest of the collected kcat values $\left(450-1200 \mathrm{~s}^{-1}\right)$ and was the twelfth most expressed protein in the Caco-2 transporter proteome (2700 fmol/mg total protein)(Figure 2AB). GLUT1 thus represents the high extreme of transport capacity $\left(V_{\max }\right)$, but its narrow 
substrate specificity suggests that it has limited relevance for drug molecules [online databases list glucose, galactose, mannose and glucosamine as substrates; http://slc.bioparadigms.org)] [19].

By contrast, several antibiotic and antiviral drugs are substrates for the oligopeptide transporter 1 (PEPT1/SLC15A1) [2, 20-22]. The lower kcat and expression levels combine to give a $V_{\max }$ approximately $1 / 200$ that of GLUT1. Notably, PEPT1 is still considered a highcapacity drug transporter as other transporters with known drug substrates typically exhibit $V_{\max }$ values two or more orders of magnitude lower [23-25]. Thus, PEPT1 represents the highcapacity end of transporters with proven relevance for drugs.

A hallmark of carrier-mediated transport is its adherence to saturable, MichaelisMenten-type kinetics (Box 2). To illustrate the effect of saturable transporter kinetics, we compared the rates that would result from carrier-mediated transport with observed transepithelial permeabilities, at two different substrate concentrations. We assumed a Michaelis constant $\left(K_{m}\right.$; Box 2$)$ of $50 \mu \mathrm{M}$, corresponding to the average of more than 700 values for drugs and drug-like compounds collected from the literature (Figure 2C). At $50 \mu \mathrm{M}$ substrate concentration - i.e., when carrier-mediated transport rates are half of the $V_{\max }-$ the extraordinary parameters for GLUT1 resulted in a rate almost eight times higher than that observed experimentally for the high-permeability drug verapamil in Caco-2 monolayers. By contrast, the corresponding parameters for the low-affinity/high-capacity transporter PEPT1 cannot explain the observed rates for verapamil or propranolol (which exceeded the PEPT1mediated rates by more than 20 -fold and fourfold, respectively)(Figure $3 \mathrm{Ai}$ ).

Adherence to Michaelis-Menten kinetics dictates that when the substrate concentration is increased from $1 \times K_{m}$ to $10 \times K_{m}$ (i.e., to $500 \mu \mathrm{M}$ in this example; Figure 3 Aiii), carriermediated rates increase by approximately 1.8 times. Passive transmembrane diffusion rates, on the other hand, increase linearly (i.e., tenfold). At the higher concentration, the rate 
mediated by GLUT1 was thus only 1.4 times higher than the transepithelial verapamil rate; PEPT1 mediated a rate less than $1 \%$ of that observed for verapamil (Figure 3Aiii).

Arguably, low-affinity (high- $K_{m}$ ) transporter substrates may not always reach saturation within relevant concentration ranges, making it difficult to detect carrier-mediated transport processes. However, a necessary consequence if $K_{m}$ exceeds the substrate concentration is that only a fraction of the total transporter capacity is used (illustrated using a $K_{m}$ of $500 \mu \mathrm{M}$ in Figures 3Ai and 3Aiii). Consequently, while low-affinity substrates may escape detection in typical transport experiments (Box 2), they are also less likely to reach the rates observed for highly permeable drugs.

Notably, for rapidly transported compounds, in vitro permeability is limited by the diffusion rate through the unstirred water layer adjacent to the cell surface $[11,16,26]$. When this in vitro artifact is considered, several-fold higher permeabilities are observed [12]. Thus, the efficiency of involved transporters needs to be even higher than that suggested above to explain the transport of high-permeability drugs. Further, more than $40 \%$ of marketed oral drugs - including verapamil - give intestinal concentrations higher than $500 \mu \mathrm{M}$ after oral dosing (and more than 30\% higher than $1 \mathrm{mM}$ ), making the demands on active transport efficiency far higher than what would be explained by the observed kinetic parameters [27].

Measured kcat values for organic substrates were only available for a few transporters (Figure 2B). What transport rates could we expect from the other transporters that are expressed in Caco-2 cells? An educated guess can be made by assuming that all transporters have kcat values from the same distribution as the collected literature values. By randomizing a kcat value from this distribution, and multiplying it with a randomly selected expression level (from the global proteomics measurements; Figure 2A) we obtained a hypothetical, yet relevant transporter $V_{\max }$. By repeating this procedure 10,000 times, we obtained a statistical distribution reflecting the likelihood of observing a certain $V_{\max }$ value. For each of these 
10,000 cases, we randomly selected a $K_{M}$ from the experimentally observed parameter distribution (Figure 2C), and calculated the transport rates expected at 50 and $500 \mu \mathrm{M}$ substrate concentrations.

The resulting rates are shown in Figure 3Aii and 3Aiv. These were calculated assuming that substrate transport was mediated by a single transporter (shown in green) or five (orange) or ten (red) transporters, all randomly selected from the observed parameter distributions. It was apparent that only in rare cases could a single transporter mediate the high rates observed for verapamil transport. For a 50 micromolar- $K_{M}$ substrate, the observed verapamil rate at $50 \mu \mathrm{M}$ concentration was reached in $7 \%$ of 10,000 randomized cases (Figure 3Aii). At $500 \mu \mathrm{M}$, the same was true in $1.3 \%$ of all cases (Figure 3Aiv). Propranolol's more moderate permeability was reached in 18 and $7 \%$ of all cases (at 50 and $500 \mu \mathrm{M}$, respectively).

Naturally, these numbers would increase if several transporters in parallel were to mediate substrate flux. Still, with panels of five transporters, only $35 \%$ (at $50 \mu \mathrm{M}$ ) and less than $8 \%$ (at $500 \mu \mathrm{M}$ ) of 10,000 such cases gave rates that could explain the measured verapamil permeability. The corresponding numbers for ten-transporter panels were $63 \%$ (at $50 \mu \mathrm{M})$ and $18 \%(500 \mu \mathrm{M})$.

Thus, the high transepithelial permeabilities observed for many completely absorbed oral drugs cannot, as a rule, be explained by transport via a single carrier. Only the rare combination of a highly expressed transporter with a high intrinsic transport capacity, or a large panel of moderate-to-high capacity transporters would allow the necessary transport rates. 


\section{Can transporters alone explain observations that define passive drug permeability?}

When passive transmembrane diffusion dominates the transport across a cell monolayer, transport rates are expected to increase linearly with concentration, and rates across a cell monolayer will be equal regardless of the transport direction [16] (Box 2). This behavior is often observed in vitro, and linear absorption increases are common in dose escalation studies of orally administered drugs [28-30]. However, the absence of such concentration dependence and/or direction dependence does not rule out transporter involvement. If the $K_{m}$ is high compared with the range of drug concentrations tested, saturation will not be observed. Correspondingly, direction-dependent transport across a cell monolayer would not be expected if the transporters (in both the apical and basolateral membranes) are equilibrative, that is, they mediate transport along substrate concentration gradients (Box 1). The question is, then, if we assume that transmembrane diffusion is negligible, and that all transport is carrier mediated, what is the likelihood of observing transport directionality and transportconcentration profiles that are consistent with passive transmembrane diffusion?

We sought to answer this with the procedure outlined in Figure 1B. Drug permeability across a cell monolayer was simulated using a three-compartment model (analogous to, for example, [31, 32]) (Box 2). The simulations were performed in the absence of passive transmembrane diffusion (i.e., in line with the transporters-only hypothesis) using the collected transporter $K_{M}$, kcat and expression level data to define carrier-mediated transport processes. Each simulation was performed in both directions across the cell layer, and repeated at twenty concentrations covering six orders of magnitude (10 nM-10 mM).

We explored three different scenarios: (1) randomizing the parameters for each transporter independently (corresponding to distinct transporter panels in the apical and basolateral membranes and, in each membrane, different parameters depending on the transport direction - i.e., unidirectional transporters); (2) randomizing transporters in the two 
membranes separately but with each transporter having identical parameters in either direction (corresponding to distinct but bidirectional equilibrative transporter panels in each membrane); and (3) using a combination of unidirectional and bidirectional transporters in each cell membrane.

We also wanted to assess whether multiple transporters in each membrane would result in concentration and/or direction independence (mimicking a passive transmembranediffusion scenario), since in theory drugs could be transported by as yet unidentified carriers. Most marketed drugs target between one and eight distinct proteins $\left(5^{\text {th }}\right.$ to $95^{\text {th }}$ percentile range) [33]. Assuming a greater promiscuity toward transporters, we randomized 1000 transporter panels for each scenario, each comprising one, five or ten transporters in each membrane and transport direction.

We used three criteria to evaluate how well the simulated transport matched the expectations for passive transmembrane diffusion: first, the rate-versus-concentration profiles should have a good fit to a linear model (signified by linear $r^{2}>0.9$ ); second, this fit to a linear model should be better than that to a Michaelis-Menten model (signified by a higher model probability based on the Akaike information criterion); and third, rates in one direction across the cell layer should, at all concentrations, be within 1.25 times the rates in the opposite direction (including a margin of error to account for experimental uncertainties).

Our simulations for Scenario 1 (i.e., independent randomization of transporter panels) showed a good linear fit in 92 of the 3000 simulated cases (3\%) (Figure 3B). However, only one single case had a better fit to a linear relationship than to the Michaelis-Menten model. Visual inspection of the rate-versus-concentration profiles revealed marked curvature in most of the 92 potentially linear cases (see red lines in Figure 3C for examples). Thus, rate-versusconcentration profiles that could be mistaken for passive transmembrane diffusion were extremely rare: even a permissive cut-off value resulted in less than five potential linear cases 
among the 3000 simulated ones. As would be expected from the Michaelis-Menten equation, the near-linear cases were defined by $K_{m}$ values that were high compared to the examined concentration range. All cases with linear $r^{2}>0.9$ had apical uptake transporter $K_{m}>$ $2,400 \mu \mathrm{M}$; the one case with a better fit to the linear model had a $K_{m}$ of $15,500 \mu \mathrm{M}$. Millimolar-range $K_{m}$ values for drug molecules have, to our knowledge, been reported only for PEPT1 (with values ranging between 4 and 8,600 $\mu \mathrm{M}$ in our collected literature data)[3437].

Similar results were observed in Scenario 2 (separate panels of bidirectional transporters in the two membranes) and Scenario 3 (combinations of unidirectional and bidirectional transporters), with $138(4.6 \%)$ and $130(4.3 \%)$ of the 3000 cases showing linear $r^{2}>0.9$. Only one of these had a better fit to the linear than to the Michaelis-Menten model (Figure 3B). Notably, most of these near-linear cases referred to simulations with a single transporter in each membrane and direction. Our analyses thus suggest that multitransporter substrates have a lower likelihood of exhibiting a linear concentration dependence than do transporterselective substrates.

As for the concentration-independence criteria, the direction-independence criterion also suggests that observations of 'passive transmembrane diffusion' would be limited to specific circumstances if all transport were carrier mediated. In the independently randomized (unidirectional-transporter) Scenario 1, none of the simulations resulted in a flux ratio close to unity (Figure 3B). Observations of direction-independent rates were more common in the bidirectional transporter Scenario 2 (476 cases; $16 \%$ of the simulations), while the mixedmechanism Scenario 3 gave results between the two 'pure' scenarios (43 directionindependent cases; 1.4\%).

For comparison, we also performed simulations of an extreme scenario with identical bidirectional transporter panels in the two cell membranes (meaning that either each 
transporter is expressed in both membranes or there is another transporter in the opposing membrane that has the same $V_{\max }$ and $K_{m}$ ). While this scenario is highly unlikely given available subcellular localization data (Figure 2A), it allowed us to explore the limits of carrier-mediated transport. As expected, all 3000 such simulations resulted in flux ratios equal to one, while transport linearity was observed in a comparable number of cases as in the physiologically more plausible Scenarios 1-3 (linear $r^{2}>0.9$ in 170 cases; 5.7\%).

\section{Concluding remarks}

In conclusion, experimental observations consistent with passive transmembrane diffusion are not possible in the transporters-only model, unless the involved transporters fulfill specific criteria. The transporters would need to be highly efficient to account for the rates observed for high-permeability compounds. Unless the compounds 'get stuck' inside the cell, such transporters would be needed on both sides of the cellular barrier (e.g., in the apical and basolateral membranes of intestinal epithelial cells). For the transporters-only model to explain observations of linear concentration-dependence, the involved transporters must not be saturated within relevant concentration ranges (i.e., beyond low-millimolar concentrations for some orally dosed drugs). Finally, direction-independent transport rates imply nearidentical transporter parameters in both membranes and both directions (i.e., either identical transporters, or different transporters with very similar drug transport properties). Importantly, these criteria need to be fulfilled for all drug molecules that show direction- and concentration-independent permeability - regardless of their chemical structures, physicochemical properties and observed transport rates.

While our results do not completely refute the transporters-only hypothesis, they demonstrate that it is unlikely for transporters alone to explain most observations in transcellular drug transport, unless the transporters involved have very different 
characteristics from those of the solute carrier (SLC) and ATP-binding cassette (ABC) transporters currently known to translocate drug molecules. Currently there are no experimental data to support the transport of many (or possibly all) drug molecules via other classes of transporters. One avenue to identify such novel drug transporters would be the use of genome-wide single-gene knockout libraries in model organisms like Saccharomyces cerevisiae [38, 39], CRISPR-Cas9 knock-out libraries in human cells [40, 41], or human haploid genetic screens [42]. These techniques have been used to screen for proteins that alter cellular drug-resistance patterns - an indication of altered drug exposure. It will be interesting to see whether ongoing proteome-wide screening efforts identify new transporters that fit the criteria listed above. 


\section{Acknowledgements}

The authors gratefully acknowledge the assistance from Mr. Amin Alimohammadi in the mining of literature transporter data. This work was supported by the Swedish Fund for Research without Animal Experiments, the Carl Trygger Foundation, and the Swedish Research Council (grants no. 9478 and 21386). Luca A. Fenu was supported by the RPF Postdoctoral Fellowship Program from F. Hoffmann-LaRoche, Basel, Switzerland. 


\section{References}

1. Giacomini, K.M., et al. (2010) Membrane transporters in drug development. Nat. Rev. Drug Discov. 9, 215-236.

2. Hillgren, K.M., et al. (2013) Emerging transporters of clinical importance: an update from the International Transporter Consortium. Clin. Pharmacol. Ther. 94, 52-63.

3. Di, L., et al. (2012) Evidence-based approach to assess passive diffusion and carriermediated drug transport. Drug Discov. Today 17, 905-912.

4. Sugano, K., et al. (2010) Coexistence of passive and carrier-mediated processes in drug transport. Nat. Rev. Drug Discov. 9, 597-614.

5. Dobson, P.D. and Kell, D.B. (2008) Carrier-mediated cellular uptake of pharmaceutical drugs: an exception or the rule? Nat. Rev. Drug Discov. 7, 205-220.

6. Balaz, S. (2012) Does transbilayer diffusion have a role in membrane transport of drugs? Drug Discov. Today 17, 1079-1087.

7. Smith, D., et al. (2014) Passive lipoidal diffusion and carrier-mediated cell uptake are both important mechanisms of membrane permeation in drug disposition. Mol. Pharmaceut. 11, 1727-1738.

8. Kell, D.B. (2014) What would be the observable consequences if phospholipid bilayer diffusion of drugs into cells is negligible? Trends Pharmacol. Sci. DOI: 10.1016/j.tips.2014.10.005

9. Di, L., et al. (2009) Drug-like property concepts in pharmaceutical design. Curr. Pharm. Des. 15, 2184-2194.

10. Waring, M.J. (2010) Lipophilicity in drug discovery. Expert Opin. Drug Discov. 5, 235-248. 
11. Artursson, P., et al. (1996) Caco-2 monolayers in experimental and theoretical predictions of drug transport. Adv. Drug Deliv. Rev. 22, 67-84.

12. Avdeef, A., et al. (2005) Caco-2 permeability of weakly basic drugs predicted with the double-sink PAMPA pKa(flux) method. Eur. J. Pharm. Sci. 24, 333-349.

13. Engman, H., et al. (2003) Enantioselective transport and CYP3A4-mediated metabolism of R/S-verapamil in Caco-2 cell monolayers. Eur. J. Pharm. Sci 19, 5765.

14. Wisniewski, J.R., et al. (2012) Extensive quantitative remodeling of the proteome between normal colon tissue and adenocarcinoma. Mol. Syst. Biol. 8, 611.

15. Artursson, P. (1990) Epithelial transport of drugs in cell culture. I: A model for studying the passive diffusion of drugs over intestinal absorptive (Caco-2) cells. $J$. Pharm. Sci. 79, 476-482.

16. Hubatsch, I., et al. (2007) Determination of drug permeability and prediction of drug absorption in Caco-2 monolayers. Nat. Protocols 2, 2111-2119.

17. Carruthers, A., et al. (2009) Will the original glucose transporter isoform please stand up! Am. J. Physiol. Endocrinol. Metabol. 297, E836-848.

18. Ferraris, R.P. and Diamond, J. (1997) Regulation of intestinal sugar transport. Physiol. Rev. 77, 257-302.

19. Mueckler, M. and Thorens, B. (2013) The SLC2 (GLUT) family of membrane transporters. Mol. Asp. Med. 34, 121-138.

20. Hironaka, T., et al. (2009) Quantitative Evaluation of PEPT1 Contribution to Oral Absorption of Cephalexin in Rats. Pharm. Res. 26, 40-50.

21. Posada, M.M. and Smith, D.E. (2013) Relevance of PepT1 in the intestinal permeability and oral absorption of cefadroxil. Pharm. Res. 30, 1017-1025. 
22. Yang, B., et al. (2013) Impact of peptide transporter 1 on the intestinal absorption and pharmacokinetics of valacyclovir after oral dose escalation in wild-type and PepT1 knockout mice. Drug Metab. Dispos. 41, 1867-1874.

23. Ozawa, N., et al. (2004) Transporter database, TP-Search: a web-accessible comprehensive database for research in pharmacokinetics of drugs. Pharm. Res. 21, 2133-2134.

24. Morrissey, K.M., et al. (2012) The UCSF-FDA TransPortal: a public drug transporter database. Clin. Pharmacol. Ther. 92, 545-546.

25. Smith, D.E., et al. (2013) Proton-coupled oligopeptide transporter family SLC15: physiological, pharmacological and pathological implications. Mol. Asp. Med. 34, 323-336.

26. Wilson, F.A., et al. (1971) Unstirred water layers in intestine: rate determinant of fatty acid absorption from micellar solutions. Science 174, 1031-1033.

27. Benet, L.Z., et al. (2011) BDDCS applied to over 900 drugs. AAPS J. 13, 519-547.

28. Hayeshi, R., et al. (2008) Comparison of drug transporter gene expression and functionality in Caco-2 cells from 10 different laboratories. Eur. J. Pharm. Sci. 35, 383-396.

29. Kikuchi, R., et al. (2013) In vitro P-glycoprotein efflux ratio can predict the in vivo brain penetration regardless of biopharmaceutics drug disposition classification system class. Drug Metab. Dispos. 41, 2012-2017.

30. Polli, J.W., et al. (2001) Rational use of in vitro P-glycoprotein assays in drug discovery. J. Pharmacol. Exp. Ther. 299, 620-628.

31. Tachibana, T., et al. (2010) Model Analysis of the Concentration-Dependent Permeability of P-gp Substrates. Pharm. Res. 27, 442-446. 
32. Korjamo, T., et al. (2007) Decrease in Intracellular Concentration Causes the Shift in Km Value of Efflux Pump Substrates. Drug Metab. Dispos. 35, 1574-1579.

33. Rask-Andersen, M., et al. (2011) Trends in the exploitation of novel drug targets. Nat. Rev. Drug Discov. 10, 579-590.

34. Anderle, P., et al. (2006) Genetic variants of the human dipeptide transporter PEPT1. J. Pharmacol. Exp. Ther. 316, 636-646.

35. Tsuda, M., et al. (2006) Transport characteristics of a novel peptide transporter 1 substrate, antihypotensive drug midodrine, and its amino acid derivatives. $J$. Pharmacol. Exp. Ther. 318, 455-460.

36. Ogihara, T., et al. (2009) Oseltamivir (tamiflu) is a substrate of peptide transporter 1. Drug Metab. Dispos. 37, 1676-1681.

37. Balimane, P. and Sinko, P. (2000) Effect of ionization on the variable uptake of valacyclovir via the human intestinal peptide transporter (hPepT1) in CHO cells. Biopharm. Drug Dispos. 21, 165-174.

38. Lum, P.Y., et al. (2004) Discovering modes of action for therapeutic compounds using a genome-wide screen of yeast heterozygotes. Cell 116, 121-137.

39. Giaever, G., et al. (2002) Functional profiling of the Saccharomyces cerevisiae genome. Nature 418, 387-391.

40. Wang, T., et al. (2014) Genetic screens in human cells using the CRISPR-Cas9 system. Science 343, 80-84.

41. Shalem, O., et al. (2014) Genome-scale CRISPR-Cas9 knockout screening in human cells. Science 343, 84-87.

42. Winter, G.E., et al. (2014) The solute carrier SLC35F2 enables YM155-mediated DNA damage toxicity. Nat. Chem. Biol. 10, 768-773. 
43. Schlessinger, A., et al. (2010) Comparison of human solute carriers. Protein Sci. 19, 412-428.

44. Hagenbuch, B. and Stieger, B. (2013) The SLCO (former SLC21) superfamily of transporters. Mol. Asp. Med. 34, 396-412.

45. Uhlen, M., et al. (2010) Towards a knowledge-based Human Protein Atlas. Nat. Biotechnol. 28, 1248-1250. 


\section{Box 1. Families of drug transporters}

Two major gene families are known to be involved in the cellular transport of drug molecules: the ATP-Binding Cassette (ABC) and the Solute Carrier (SLC) superfamilies. ABC transporters catalyze the hydrolysis of ATP to drive substrate translocation and typically mediate efflux; that is, substrate transport from the cell interior to the exterior. The SLC family is more diverse in both protein structure and transport mechanism [43]. Some SLCs (including PEPT1/SLC15A1 and NTCP/SLC11A1) are driven by the co- or countertransport of inorganic ions (e.g., $\mathrm{H}^{+}, \mathrm{Na}^{+}, \mathrm{Cl}^{-}$) or small organic molecules (e.g., OAT1/SLC22A6, a glutarate/ketoglutarate exchanger). The concentration gradients of the coupled ions/molecules are typically maintained by other channels/transporters, such as $\mathrm{Na}^{+} / \mathrm{K}^{+}$-ATPases. Such SLC transporters are thus indirectly coupled to ATP hydrolysis, and can mediate concentrative transport; that is, transport of the primary substrate against a concentration gradient. Other SLC transporters (including the SLC2 glucose transporters; e.g., GLUT1/SLC2A1) are equilibrative (or facilitative); that is, transport occurs through facilitated diffusion along the substrate concentration gradient. Notably, the mechanism remains unknown for several transporter families. This includes the important drug transporter family SLCO/OATP [44]. 


\section{Box 2. How cellular drug transport is measured and transporter effects are inferred}

The most commonly used methods to study drug transport in vitro can be divided conceptually into assays for cellular drug uptake and assays for drug permeability. The first type measures the rate at which a drug added to the cell exterior is accumulated within the cell; this method is applicable both to cells in suspension and to adherent cells. By contrast, the latter assay type measures the rate of transport across the cell; it requires cells that form a tight monolayer when cultured on semiporous filters, such as Caco-2 or Madine-Darby Canine Kidney (MDCK) cells.

Various approaches are used to assess carrier-mediated transport in both assays, all with different caveats. Uptake (or permeability) is often determined in a cell type that overexpresses one or more specific transporters. If such measurements differ significantly from similar measurements in a control cell line (typically one transfected with an empty expression vector), it is inferred that the transport is carrier-mediated. Other options include comparing transport rates in the absence and presence of a compound known to inhibit the transporter of interest. This inhibitor approach is limited because most known transport inhibitors lack specificity (i.e., it is often difficult to pinpoint the transporters involved) and few or no inhibitors are available for less characterized transporters (i.e., there may be residual carrier-mediated flux even when inhibitors are present, and lack of inhibition does not necessarily mean that transporters are not involved).

A more reliable indicator of carrier-mediated transport is an observable concentration dependence of the transport rates. Carrier-mediated transport follows saturable MichaelisMenten kinetics: at low concentrations, transport rates increase approximately linearly with concentration. At higher concentrations the relationship becomes increasingly nonlinear, with 
transport rates increasing asymptotically towards a maximum transport rate $\left(V_{\max }\right)$ that is limited by the number of transporter molecules expressed in the cell membrane and the intrinsic capacity of each transporter to translocate its substrate (described by the turnover number, kcat). The extent of saturation is determined by the Michaelis constant $\left(K_{M}\right)$; that is, the concentration where transport occurs at one-half of $V_{\max }$.

An alternative to concentration-dependent rate measurements is to determine transport rates in both directions across a cell monolayer. These rates are used to calculate flux ratios (typically expressed as efflux ratios; i.e., the ratio of basolateral-to-apical rates over rates in the opposite direction). Another clear indicator of transporter involvement is when transport rates differ depending on which side of the monolayer the drug is applied (i.e., non-uniform flux ratios). Importantly, however, equilibrative transporters (which mediate substrate flux along concentration gradients; Box 1) can - under certain circumstances (see Figure 3B in main text) - give rise to direction-independent rates. Thus, near-unity flux ratios do not unambiguously exclude transporter involvement. If the bidirectional permeability experiment is performed over a broad concentration range, the chances of identifying equilibrative transporters increase since flux ratios start to differ from 1 when the transporters in one membrane reach saturation. Direction-independent permeability has been demonstrated for numerous compounds (e.g., [28-30]); most studies have been performed at one or two concentrations and additional experiments are thus warranted to confirm directionindependence over wider concentration ranges. 


\section{Figure legends}

Figure 1. Assessing proposed mechanisms for cellular drug permeability using a systems

biology approach. A. In the predominant view, drug transport across cell membranes is a combination of passive transmembrane diffusion and carrier-mediated transport ("coexistence model'). Depending on the molecule's chemical structure, either transport mechanism may dominate the overall transport. The alternative hypothesis states that transmembrane diffusion is negligible for drug molecules; instead, transport is exclusively carrier-mediated ('transporters-only model'). It is unclear whether this latter model is compatible with experimental observations typical for passive transcellular permeability (i.e., concentrationindependent and direction-independent permeability). B. A systems-biology approach to test the transporters-only hypothesis. Transcellular drug permeability was simulated in the absence of passive transmembrane diffusion. Relevant transporter parameters were obtained from global proteomics measurements of transporter expression in the monolayer-forming intestinal epithelial cell line Caco-2 and through literature mining for drug-transport kinetics measurements (1). Transport was explored using different transport mechanisms (concentrative and equilibrative transporters) and different numbers of contributing transporters (2). The resulting permeability profiles were evaluated to see under which circumstances the results supported a transporters-only model (3); that is: (a) a linear rateversus concentration relationship; ( b) a linear fit relationship better than a saturable Michaelis-Menten model; (c) approximately equal transport rates in either direction across the cell layer.

Figure 2. Transporter properties used in the simulations of cell permeability. A. Expression of transporters in Caco-2 intestinal epithelial cells. Data are presented as means (dark blue bars) and standard deviations (light blue bars). The facilitative glucose transporter 
GLUT1 (SLC2A1) and the oligopeptide transporter PEPT1 (SLC15A1) are highlighted because they were used as examples in further analyses. Subcellular localization data were collected from human intestinal samples in the Human Protein Atlas (http://www.proteinatlas.org/) [45] and the Bioparadigms SLC Tables (http://slc.bioparadigms.org) [19]. Proteomic expression data are reused, with permission, from [14]. B. Turnover numbers (kcat) collected from the literature. Reports were included that refer to eukaryotic solute carrier (SLC) or ATP-binding cassette (ABC) superfamily transporters having organic substrates with molecular weights in excess of $50 \mathrm{Da}$. In general, the kcat values refer to endogenous substrates (or close derivatives). It is reasonable to assume that drug molecules are not transported as efficiently as endogenous substrates. C. Distribution of $K_{M}$ values for drugs and drug-like compounds transported by human SLC and ABC superfamily transporters. Data collected from the literature $(n=445$ and 293 for SLC and $\mathrm{ABC}$ transporters, respectively).

Figure 3. Testing the transporters-only hypothesis. A. Comparison between predicted carrier-mediated transport rates and observed transcellular drug permeabilities. The top row shows the transport rates expected at a substrate concentration of $50 \mu \mathrm{M}$ (i-ii), and the bottom row at $500 \mu \mathrm{M}$ (iii-iv). In each row, the plot on the left (i and iii) shows the rates expected for substrates of the facilitative glucose transporter 1 (GLUT1/SLC2A1) (blue lines) and the di/tripeptide transporter 1 (PEPT1/SLC15A1) (red lines). Unbroken and broken lines represent substrates with $K_{M}$ values of 50 and $500 \mu \mathrm{M}$, respectively. For comparison, measured permeability coefficients (Papp) for the highly permeable drugs verapamil and propranolol were converted to the corresponding transport rates (black circles and squares, respectively). The panels on the right (ii and iv) show the carrier-mediated rates that would result from one (green), five (orange) or ten (red) transporters, each with expression levels 
and kcat values randomly selected from the distributions in Figure 2. Unbroken lines show the median transport rate calculated for 10,000 randomized transporters. Shaded areas show the rate interval covered by the randomized cases, with darker color indicating a greater likelihood of observing a certain rate. B. Fulfillment of passive-diffusion criteria in transporters-only simulations. Three scenarios were simulated: (1) independently randomized unidirectional, concentrative transporters; (2) bidirectional equilibrative transporters; and (3) combination of unidirectional and bidirectional transporters. The resulting transcellular permeabilities were evaluated according to three criteria consistent with passive transmembrane diffusion. These criteria were: (a) that the rate-versus-concentration relationship should have linear $r^{2}>0.9$; (b) that the fit should be better to a linear model than to a Michaelis-Menten model; and (c) the permeability should be approximately direction independent. If the transporters-only hypothesis is correct, it must account for the many experimental observations that follow these criteria. Circles show the number of simulated cases (from among 3000 cases per scenario; 1000 each with one, five, or ten transporters per membrane) that fulfil each criterion. C. Examples of transport concentration dependence. Black symbols show the rate-concentration dependence for one of only two cases among the 3000 simulations that had a better fit to a linear than to a Michaelis-Menten model. Blue symbols show random examples of simulations with a poor linear fit $\left(r^{2}<0.5\right)$. Red symbols show the range of cases with linear $r^{2}>0.9$, but where the Michaelis-Menten model gave a better fit than a linear model. 


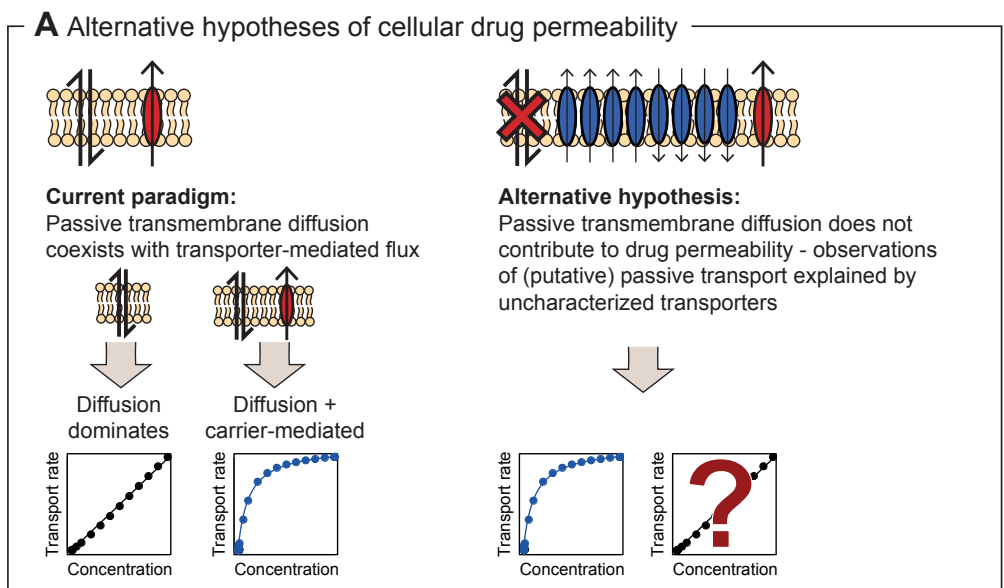

- B Simulation approach to assess the alternative 'transporters-only' hypothesis

1. Literature mining for relevant parameters

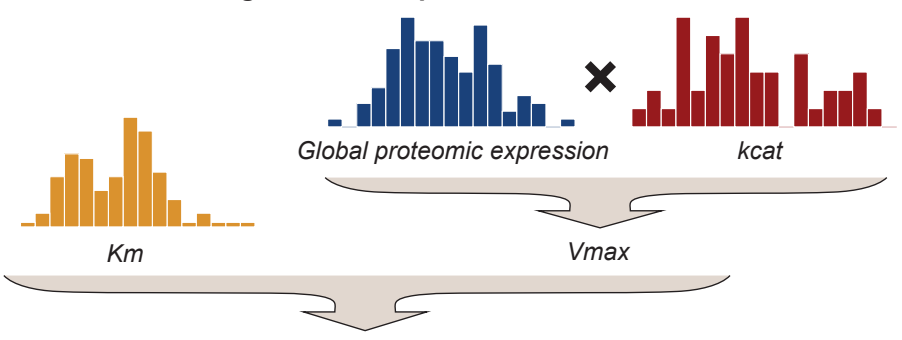

2. Simulation of example cases in the absence of passive diffusion ( $n=1000$ for each scenario)

Scenario 1:

Transporter parameters randomized independently

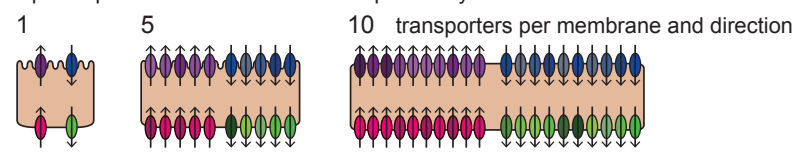

Scenario 2:
Each transporter has identical parameters in both directions (i.e., equilibrative transporters)

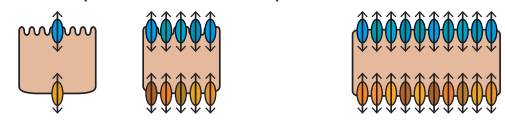

Scenario 3:

Combination of unidirectional and bidirectional transporters
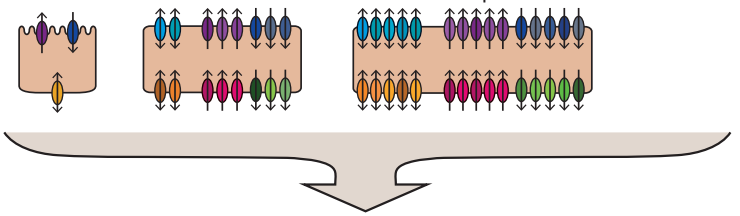

3. Evaluation of the fit to the alternative hypothesis

Criterion A: Good linear fit $\left(\mathrm{r}^{2}>0.9\right)$
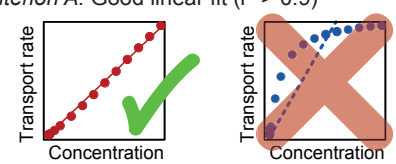

Criterion B: Better fit to linear model than to saturable Michaelis-Menten mode
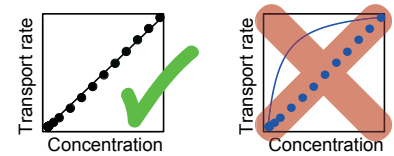

Criterion $C$ : Near-equal transport rate in both directions (flux ratio $\approx 1$ )
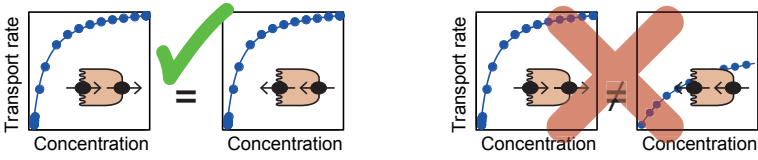


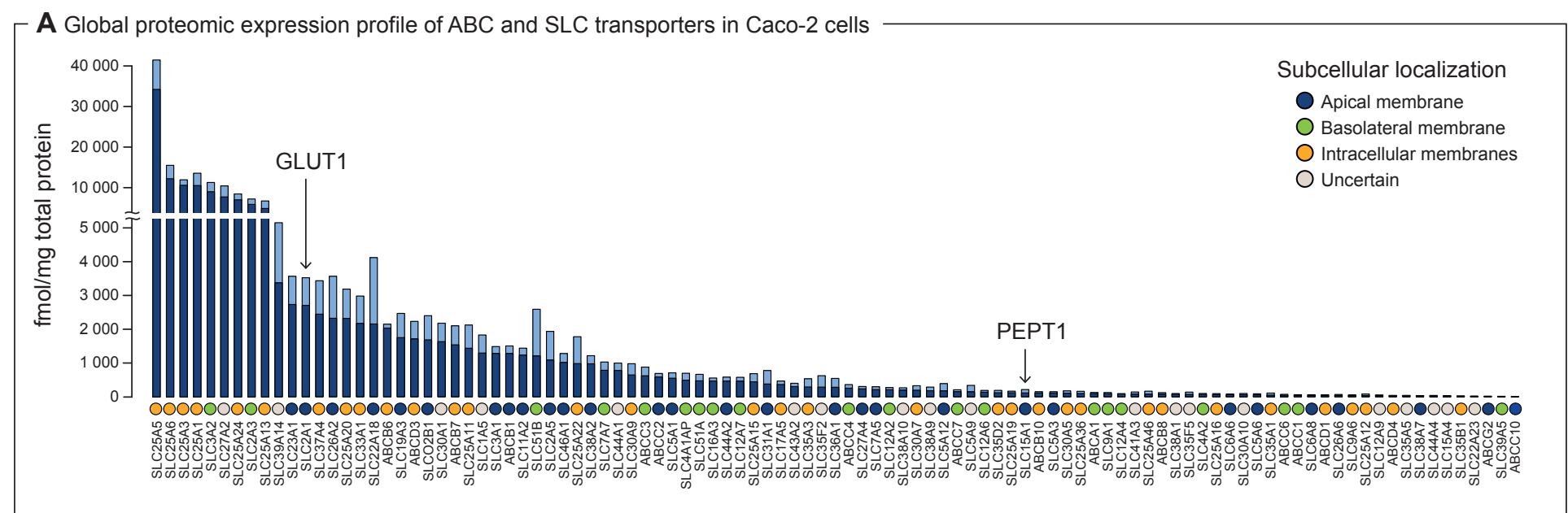

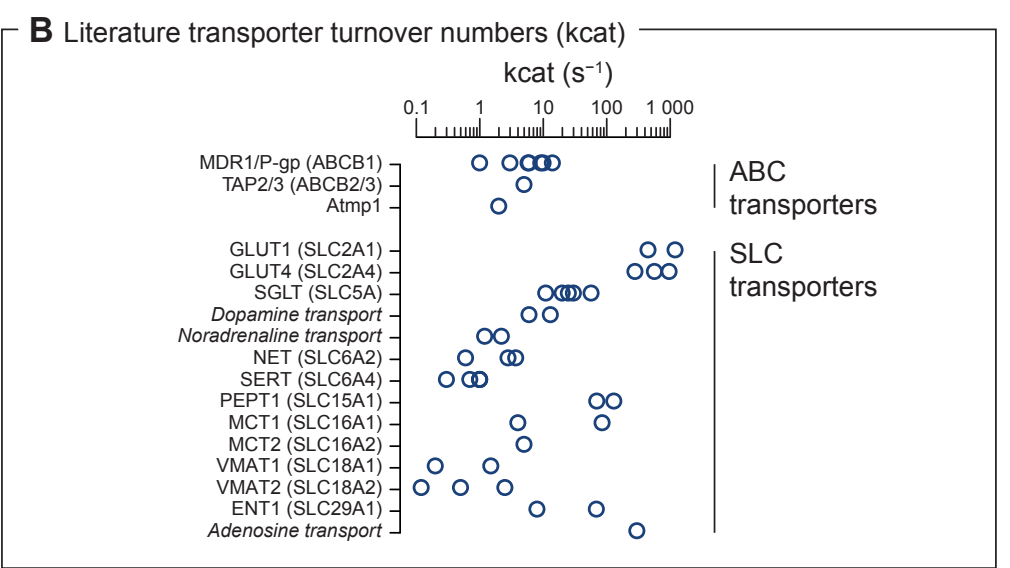

- C Literature drug-transporter $K_{M}$ data

$$
\mathrm{Km}(\mu \mathrm{M})
$$

$$
\begin{array}{lllllll}
0.001 & 0.01 & 0.1 & 1 & 10 & 100100010000100000
\end{array}
$$

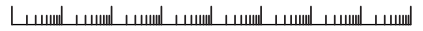

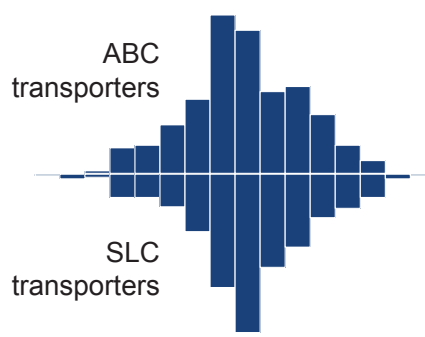


A Comparison of transporter-mediated and passive diffusion flux rates

Transport rates at $50 \mu \mathrm{M}$

i $\quad \begin{aligned} \text { GLUT1 } & \text { GLUT1 } \\ \left(K_{M}=50 \mu \mathrm{M}\right) & \left(K_{M}=500 \mu \mathrm{M}\right)\end{aligned}$

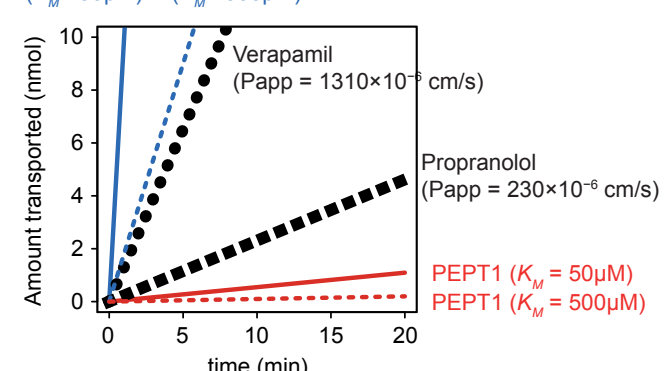

ii

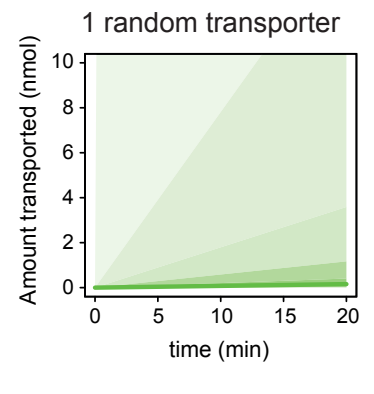

5 random transporters 10 random transporters
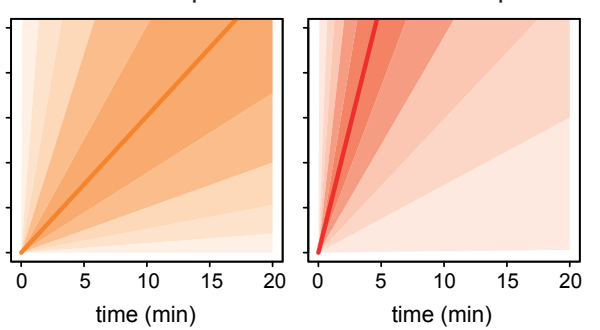

Transport rates at $500 \mu \mathrm{M}$

iii

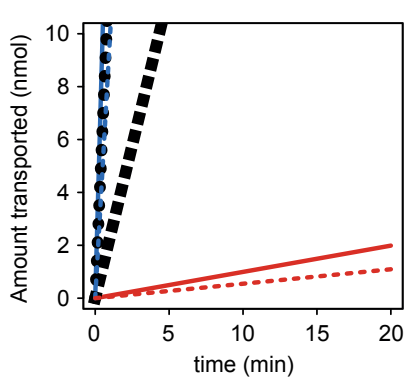

1 random transporter 5 random transporters 10 random transporters

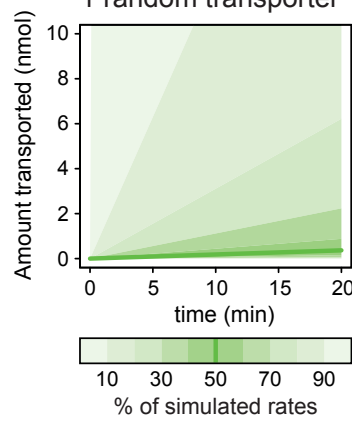

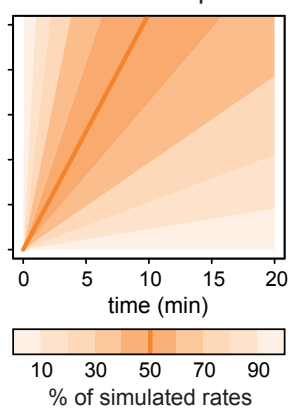

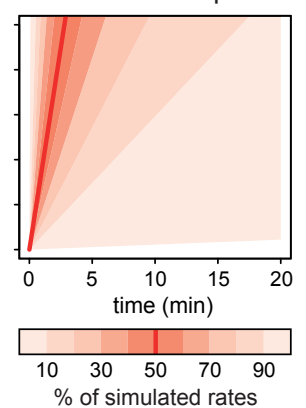

B Fulfillment of passive-diffusion criteria in transporters-only simulations

Scenario 1:

Independent transporter panels

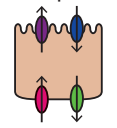

Scenario 2:

Identical transport parameters in both directions

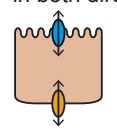

Scenario 3:

Combination of unidirectional and bidirectional transporters [on
C Example simulated transport rates as a function of drug concentration

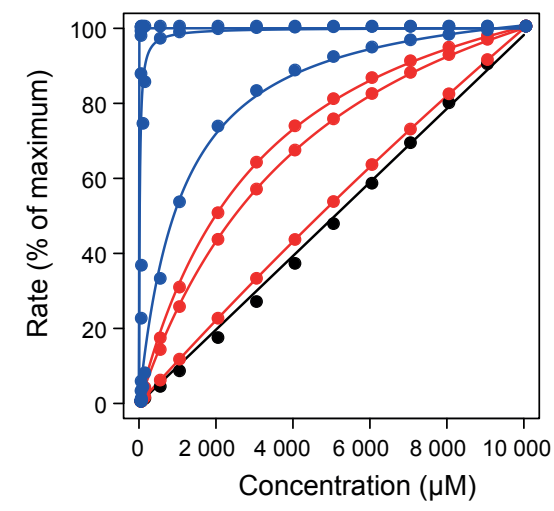

- Simulations with linear $r^{2}>0.9$; better fit to linear than to Michaelis-Menten model - Simulations with linear $r^{2}>0.9$; better fit to Michaelis-Menten than to linear model

- Simulations with linear $r^{2}<0.5$; better fit to Michaelis-Menten than to linear model 